\title{
Intercostal Artery Branch
}

National Cancer Institute

\section{Source}

National Cancer Institute. Intercostal Artery Branch. NCI Thesaurus. Code C32822.

Either of three arteries (posterior or dorsal branch, collateral intercostal and spinal) that branch off of the intercostal artery. 